\title{
Bioinformatic identification of the potential afatinib drug resistance gene BIRC5 in non-small- cell lung cancer
}

\author{
Xiaoxi Zhu \\ Jinan University \\ Yuanzhi Lu ( $\nabla$ yuanzhi.lu@jnu.edu.cn ) \\ Jinan University \\ Qiang Chen \\ Jinan University \\ Yin Li \\ Jinan University
}

\section{Research Article}

Keywords: Bioinformatic analysis, NSCLC, Afatinib resistance, BIRC5

Posted Date: April 27th, 2021

DOI: https://doi.org/10.21203/rs.3.rs-396954/v1

License: (c) (i) This work is licensed under a Creative Commons Attribution 4.0 International License. Read Full License 


\section{Abstract}

Background Drug resistance inevitably limits the efficacy of the second-generation epidermal growth factor receptor-tyrosine kinase inhibitor (EGFR-TKI) afatinib, and the underlying causes of this resistance have not been determined.

Methods In this study, we demonstrated that baculovirus IAP repeat protein 5 (BIRC5) may confer afatinib resistance in non-small-cell lung cancer (NSCLC) via multiple mechanisms based on integrative bioinformatic analyses.

Results Through bioinformatic analyses of microarray datasets, BIRC5 was overexpressed in both afatinib-resistant NSCLC cells and NSCLC tissues and was initially identified as a potential candidate gene causing poor survival in afatinib-resistant patients with NSCLC. The resistance function of BIRC5 in NSCLC was validated by performing protein/gene interactions and biological process annotation analyses and further validated by analyzing transcription factors targeting BIRC5 mRNA.

Conclusions We applied our results to NSCLC and showed that BIRC5 was an important candidate gene for afatinib resistance.

\section{Introduction}

Non-small-cell lung cancer (NSCLC) is the most prevalent subtype of lung cancer (approximately 85\%) [1], is mostly metastatic at diagnosis and represents the leading cause of cancer death worldwide [2]. Most patients are diagnosed at an advanced stage $[3,4]$. The discovery of activating mutations in EGFR and their use as predictive biomarkers to tailor patient therapy with EGFR TKIs has revolutionized the treatment of patients with advanced EGFR-mutant NSCLC [5].

Afatinib is a irreversively mutant EGFR-TKI and the first-line FDA-approved indication to treat locally advanced or metastatic NSCLC [6]. While afatinib achieves superior efficacy in progression-free survival (PFS) and overall survival (OS) compared with conventional chemotherapy in NSCLC, progression inevitably occurs after EGFR TKI treatment for acquired resistance, which presents challenges in the treatment of NSCLC $[7,8]$. The mechanisms of acquired resistance are classified into three types: acquired mutation of targetable driver genes, bypass signaling pathway activation and histological lineage-transformation [9]. Accordingly, pharmacological interception of the propensity of tumor cells by bypass signaling pathways derails their signaling or adhesion receptors and may allow the identification of novel targets for cancer therapy [10]. An understanding of the mechanistic bases for drug resistance would continue to inform the development of strategies to overcome or prevent clinical acquired resistance, thereby providing a greater therapeutic benefit for cancer patients [11].

BIRC5 (also named Survivin) is a small protein that belongs to the inhibitor of apoptosis protein family that inhibits caspases and blocks cell death. It is abundantly expressed in tumors compared with adult differentiated tissues and is associated with poor prognosis in many human neoplasms [12]. Because of 
selective expression in tumor but not normal tissues, for over a decade, BIRC5 has drawn considerable attention as a potential novel drug target in a variety of human cancers and consistently has been demonstrated to be a critical factor in tumor progression [13]. Most studies of BIRC5 have focused on sensitization to chemotherapy and radiotherapy, while the level of heterogeneity among patients with targeted drug treatment and its biological significance have not yet been comprehensively investigated.

In this work, BIRC5 was initially identified as a potential candidate in the GEO database. Then, we comprehensively searched the dataset and conducted a systematic bioinformatic analysis of potential genes promoting afatinib resistance in NSCLC. Moreover, the afatinib resistance role of BIRC5 was further validated by combining multiple tools, including protein/gene interactions, biological process annotation, and prediction of resistance mechanisms. Additionally, the transcription factor KLF7 may play an important role in this process. Our study provides a potential target and associated mechanisms of afatinib resistance and suggests that BIRC5 could be a prognostic biomarker for afatinib treatment.

\section{Materials And Methods}

\section{Microarray data}

The Gene Expression Omnibus (GEO) database is a high-throughput microarray and sequence functional genomic database (https://www.ncbi.nIm.nih.). In this study, the GSE62504 dataset consisted of 2 afatinib-resistant HCC827 replicates and 1 parental HCC827 cell line, which was the NSCLC adenocarcinoma cell line [14]. GSE75037 [15] included 83 LUAD samples and 83 matched adjacent lung samples, whereas GSE18842 contained 32 squamous cell carcinoma, 14 adenocarcinoma, and 45 adjacent lung tissues [16].

\section{Processing of microarray data}

The original microarray data files of these 3 downloaded datasets were analyzed through GEO2R (https://www.ncbi.nlm.nih.gov/geo/geo2r/). This online tool can be used to compare two groups under the same experimental settings or more sets of samples [17]. In this study, we used $P<0.05$ and Ifold change $(F C) \mid>1.5$ as the cutoff standards to define the DEGs.

\section{Functional annotation and pathway enrichment analysis}

To investigate the functional annotation of DEGs, this work applied annotation, visualization and a comprehensive database (DAVID, https://david.abcc ncifcrf.gov/) [18] to perform the gene ontology (GO) and Kyoto Encyclopedia of Genes and Genomes (KEGG) analyses, specifically GO enrichment analysis and KEGG signal pathway enrichment analysis, where $P<0.05$ was set as statistically significant.

\section{ONCOMINE and TIMER analysis}

The gene expression array dataset of ONCOMINE (www.oncomine.org) is a publicly accessible, online cancer microarray database that helps facilitate research using genome-wide expression analyses [19]. 
For DEGs, comparison between cancer specimens and normal control dataset analysis was performed.

The TIMER database was used to systematically analyze tumor-infiltrating immune cells (TIICs) in 32 cancer types using more than 10,000 samples from The Cancer Genome Atlas (TCGA) (https://cistrome.shinyapps.io/timer/) database [20]. We initially employed this database to assess differences in DEG expression levels in tumor types by using the TIMER database.

\section{GEPIA Dataset}

The Gene Expression Profiling Interactive Analysis (GEPIA) database (http://gepia.cancerpku.cn/index.html) was used to analyze the RNA sequencing expression data from 8,587 normal and 9,736 tumor tissue samples from the TCGA and GTEx projects [21]. To further verify DEGs, GEPIA was used to plot a gene expression level map between LUAD, lung squamous cell carcinoma (LUSC), and adjacent lung tissues in TCGA.

\section{Survival analysis}

Kaplan-Meier analysis (https://www.kmplot.com) is an online survival analysis tool consisting of 10,461 cancer samples (including 5143 breast cancer, 1816 ovarian cancer, 2437 lung cancer and 1065 gastric cancer patients) that can facilitate evaluation of the impact of 54,675 gene pairs on overall survival [22]. According to the expression levels of DEGs, lung cancer patients were divided into a high expression group and a low expression group, and the overall survival rate was further analyzed.

\section{GeneMANIA and Coremine's prediction function of BIRC5}

GeneMANIA (https://www.genemania.org/) was used to predict the potential function of the BIRC5 gene and to predict the functions of specific genes [23]. In addition, we further explored the biological process annotations of BIRC5 and afatinib resistance by querying the Coremine Medical online database (https://www.coremine.com/medical/).

\section{Identification of transcription factors of BIRC5}

To obtain the transcription factors that regulate the BIRC5 gene, FunRich (https://www.funrich.org) was used to identify a gene-rich transcription factor interaction network analysis tool [24].

\section{Statistical analysis}

GraphPad Prism 8 software (GraphPad Software, Inc., USA) statistical software was used to process the data, and $P<0.05$ was considered statistically significant.

\section{Results}

Identification and functional characterization of upregulated DEGs in afatinib-resistant NSCLC cells 
To identify the potential genes conferring afatinib resistance in NSCLC, we investigated GSE62504 using $|\log 2(\mathrm{FC})|>1$ and $P<0.05$ as thresholds to screen for differential genes (Fig. 1A), and a total of 1483 DEGs were screened, of which 700 DEGs were significantly upregulated and 783 DEGs were downregulated (Fig. 1B).

To characterize the functions of these significantly upregulated DEGs, GO and KEGG analyses were then performed as previously described. The top $5 \mathrm{GO}$ terms and enrichment pathways were determined, and these significantly upregulated DEGs were highly associated with cell adhesion, inhibition of apoptosis and inhibitor RNA transcription height (Fig. 1C). As shown in Fig. 1D, the DEGs that were simultaneously upregulated were also enriched in intercellular junctions and phagosomes, cell adhesion pathways, and PI3K-Akt signaling pathways.

\section{BIRC5 was identified as the gene conferring afatinib resistance in NSCLC}

Tumor gene heterogeneity is one of the determinants of drug resistance in the process of tumor treatment $[25,26]$. To screen the genes related to the occurrence of afatinib resistance, the GSE75037 dataset consisting of lung cancer and adjacent normal tissues was further searched through the GEO database. A total of 984 DEGs were screened (Fig. 2A), of which 312 genes were significantly upregulated and 672 genes were downregulated in cancer tissues (Fig. 2B). Interfering with mitotic arrest cells promoted their death, which was successful in the clinic [27]. The top GO terms and KEGG of upregulated DEGs affected nuclear division and mitosis (Fig. 2C) and the cell cycle (Fig. 2D). The gene expression control principles were complex and unique, and the integration of transcription and proteomic data provided additional information about gene expression control which cannot be obtained from single data[28]. To verify the reliability of the results, the GSE18842 datasets composed of lung cancer and normal tissues were retrieved. As the results shown in fig. 3A, it can be seen that 990 DEGs were identified, in which 402 DEGs were up-regulated and 588 DEG were significantly down-regulated (Fig. 3B). The GO terms were highly consistent to GSE75037 (Fig. 3C), while the DEGs were enriched in cell cycle pathways (Fig.

3D).Comparing the DEGs significantly upregulated in GSE62504, GSE18842 and GSE75037 (Fig. 4A), we found that 6 genes were obtained: BIRC5, minichromosome maintenance complex 4 (MCM4), secreted phosphoprotein 1 (SPP1), neurotonin $\mathrm{U}(\mathrm{NMU})$, collagen triple helix repeat contains 1 (CTHRC1), and E3 ubiquitin protein ligase (UHRF1).

\section{Validation of BIRC5 related to afatinib resistance in NSCLC}

The online databases Oncomine and GEPIA were used to further confirm correlated genes in NSCLC. To examine the change in BIRC5 expression between NSCLC and adjacent tissues, we analyzed their expression profiles in many independent bioinformatic datasets. Using Oncomine, we found that the BIRC5 transcription level in tumor tissues from the microarray dataset was significantly increased (Fig. 4B). TIMER was used to evaluate the expression level of hub genes in the TCGA database in NSCLC and normal tissues (Fig. 4C). Consistent with Oncomine analysis profiling, BIRC5 identified in NSCLC tissues was higher than that in normal tissues by TIMER. In addition, to further verify the expression level of BIRC5, we utilized another online analysis tool, GEPIA. The results revealed that BIRC5 was upregulated in 
LUAD and LUSC compared with normal tissues (Fig. 4D). Moreover, BIRC5 was related to the clinical staging of lung cancer (Fig. 4E). By using the human protein profile database, it was further verified that BIRC5 was positively expressed in NSCLC tissues and negatively expressed in normal lung tissues (Fig. $4 \mathrm{~F})$. All of the above data indicated that the upregulation of BIRC5 expression levels promoted the development and progression of NSCLC.

\section{Prognostic significance of BIRC5 expression in LUSC AND LUAD}

Furthermore, we investigated the correlation between BIRC5 overexpression at the mRNA level and prognosis by plotting and comparing the OS, FP, and PPS of LUSC and LUAD patients to healthy individuals through Kaplan-Meier plotter (Fig. 5). BIRC5 overexpression was associated with worse FP $(\mathrm{HR}=3.13$ (2.23-4.4), $\mathrm{P}<0.001)$ and OS $(\mathrm{HR}=2.42$ (1.9-3.09), $\mathrm{P}<0.001)$, which was negative in LUSC. Overall, the findings above implied that the mRNA expression of BIRC5 was remarkably correlated with LUAD patient survival rates and may be identified as a promising biomarker to predict the survival of LUAD patients.

\section{Prediction of the resistance mechanism of BIRC5 in NSCLC}

To determine whether BIRC5 functions in the drug resistance of cancer, GeneMANIA was employed. BIRC5 showed interactions with 20 proteins/genes (Fig. 6 B). A), of which 6 genes have been reported to be important in conferring drug resistance in multiple cancer types [29-34]. As shown in Fig. 6B, among the 6 genes, caspase 9 (CASP9) and kinetin family member 20A (KIF20A) were all downregulated in GSE62504, while aurora kinase B (AURKB), aurora kinase A (AURKA), cell division cycle 6 (CDC6) and Cullin 3 (CUL3) were all upregulated. Exploring transcription factors of BIRC5 may help to understand the mechanism of afatinib resistance in NSCLC. Then, Funrich was used to predict transcription factors targeting BIRC5, which determined that the transcription factor kruppel like factor 7 (KLF7) affects drug resistance in cancers $[35,36]$. Finally, the Coremine Medical database was employed to annotate the biological process of BIRC5. As shown in Fig. 6C, 10 biological processes were related to drug resistance. BIRC5 may participate in drug resistance by affecting cell viability, the cell cycle, migration and adhesion. Therefore, BIRC5 can confer resistance to afatinib in NSCLC through multiple mechanisms.

\section{Discussion}

Targeted resistance caused by target mutations and off-target mutations. Target mutations occurred when the main molecular target was mutated and did not respond to treatment. Off-target resistance was achieved by activating a signal pathway parallel to or bypassing the downstream signal of the target [37]. Activation of the EGFR alternative pathway also led to secondary drug resistance in $20 \%$ of NSCLC patients, thereby activating the same EGFR downstream effectors to promote tumor cell growth [38]. For example, Met amplification could activate the PI3k/AKT/mTOR signaling pathway [39]. Over the past 10 years, great strides have been made in defining the molecular mechanisms of EGFR TKI resistance in an effort to design rational strategies to overcome drug resistance [40]. Afatinib was originally designed to overcome the development of drug resistance to the first-generation EGFR-TKIs. Compared with gefitinib 
and erlotinib, beyond the efficacy comparable to first-generation TKls and similar drug-related safety profiles (mainly differentiating in terms of frequency) [41], afatinib was shown to be active against uncommon mutations [42]. However, approximately $60 \%$ of patients had new mutations in the drug target within 9-12 months of taking afatinib [43]. At present, high-throughput screening confers resistancerelated genes and drug design targeting and represents a new dawn in anticancer therapy. For bypass resistance, the most common strategy is to develop combination therapies that inhibit one or more downstream signaling proteins in addition to the primary target [44]. For example, the CDK4/6 inhibitor alpelisib inhibited drug resistance caused by PI3KCA mutation during treatment [45]. Herein, by combining microarray datasets retrieved from GEO and subsequent bioinformatic analyses, BIRC5 was identified and further validated as an important candidate for acquired afatinib resistance in NSCLC.

In this study, we characterized BIRC5 overexpression in afatinib-resistant cells and NSCLC tissues and predicted conferred resistance to afatinib in NSCLC through multiple mechanisms. BIRC5 plays an important role in cell division and growth via the regulation of mitosis [13]. However, the signaling aspect of BIRC5 biology is complex and incompletely understood and may also differ depending on the cellular context. Evidence to date suggests that activation of Akt/PKB and PI3K occurs upstream of many events that involve BIRC5 [46]. Furthermore, BIRC5 was reported to promote epithelial-to-mesenchymal transition (EMT), suggesting that BIRC5 may contribute to metastasis and chemoresistance [47]. BIRC5 is associated with progression and poor survival in LUAD, indicating that overexpression of BIRC5 is associated with an increased invasive phenotype and worse clinical prognosis. Based on these findings, BIRC5 is considered as a promising therapeutic target. YM155 (sepantronium bromide) was the first potent, selective small-molecule inhibitor of BIRC5 to be discovered [48]. A phase I study was conducted with YM155 in combination with erlotinib in patients with EGFR TKI refractory advanced NSCLC, suggesting that inhibiting BIRC5 is a potential therapeutic strategy for select patients with EGFR TKI refractory NSCLC [49]. Targeting BIRC5 drugs could guide the immune system to recognize and kill cancer cells and could increase BIRC5 peptide-specific CTLs to enable patients to kill cancer cells $[50,51]$. The transcription factor KLF7 of BIRC5 may be involved in afatinib resistance in NSCLC. In a previous investigation, KLF7 was widely expressed in numerous human tissues at low levels and functioned as an oncogene in some cancer types $[52,53]$. Taken together, we validated that BIRC5 was a candidate gene for afatinib resistance in NSCLC and could be a prognostic biomarker for afatinib treatment.

\section{Conclusion}

Overall, the current findings confirmed that BIRC5 may confer afatinib resistance in NSCLC via multiple mechanisms based on integrative bioinformatic analyses. However, investigations designed to elucidate the drug resistance mechanisms of BIRC5 in afatinib-resistant NSCLC models are still urgent.

\section{Abbreviations}

NSCLC, non-small cell lung cancer; epidermal growth factor receptor-tyrosine kinase inhibitor (EGFR-TKI); GEO, gene expression omnibus; DEGs, differentially expressed genes; DAVID, database for annotation, 
visualization and integrated discovery; GO, gene ontology; KEGG, kyoto encyclopedia of genes and genomes; KM plotter, Kaplan-Meier plotter ; MCM4, minichromosome maintenance complex 4; SPP1, secreted phosphoprotein 1; NMU, neurotonin $\mathrm{U} ; \mathrm{CTHRC1}$, collagen triple helix repeat contains 1;UHRF1, E3 ubiquitin protein ligase; CASP9, caspase 9; KIF20A, kinetin family member 20A; AURKB, aurora kinase B; AURKA, aurora kinase A; CDC6, cell division cycle 6; CUL3, Cullin 3; KLF7, kruppel like factor 7; CDK4/6, cyclin-dependent kinases 4/6; PI3K, phosphoinositide 3-kinase; BP, biological process; CC, cellular component; MF, molecular function.

\section{Declarations}

\section{Acknowledgments}

The authors would like to thank all publicly available data used in the present study.

\section{Authors' contributions}

Xiaoxi Zhu: Conceptualization, Formal analysis, drafting manuscript, review \& editing.Qiang Chen: Data curation. Yin Li:Visualization. Yuanzhi Lu: Conceptualization ,proof editing, funding support.

\section{Funding}

This study was supported by a grant from the National Natural Science Foundation of China (Grant No. 81572606).

\section{Availability of data and materials}

The datasets GSE62504,GSE75037 and GSE18842 analyzed during the current study are available in the GEO(https://www.ncbi.nlm.nih.gov).

\section{Ethics approval and consent to participate}

The datasets are accessible to the public, hence ethics approval is not considered required. This study did not require ethics approval.

\section{Consent for publication}

All authors support publishing.

\section{Competing interests}

The authors declare that they have no competing interests.

\section{Author details}


${ }^{a}$ Department of Oncology, First Affiliated Hospital of Jinan University, Guangzhou, 510630, China ,'Department of Clinical Pathology, First Affiliated Hospital of Jinan University, Guangzhou 510630, China .${ }^{\square}$ Corresponding author at: Department of Clinical Pathology, First Affiliated Hospital of Jinan University, Guangzhou 510630

\section{References}

[1] R.L. Siegel, K.D. Miller, H.E. Fuchs, A. Jemal, Cancer Statistics, 2021, CA: a cancer journal for clinicians 71(1) (2021) 7-33. https://doi.org/10.3322/caac.21654.

[2] S.B. Clark, S. Alsubait, Non Small Cell Lung Cancer, StatPearls, StatPearls Publishing Copyright (c) 2021, StatPearls Publishing LLC., Treasure Island (FL), 2021.https://www.ncbi.nIm.nih.gov/books/NBK562307.

[3] B.C. Bade, C.S. Dela Cruz, Lung Cancer 2020: Epidemiology, Etiology, and Prevention, Clinics in chest medicine 41(1) (2020) 1-24. https://doi.org/10.1016/j.ccm.2019.10.001.

[4] N.A. Thomas, N.T. Tanner, Lung Cancer Screening: Patient Selection and Implementation, Clinics in Chest Medicine 41(1) (2020) 87-97. https://doi.org/10.1016/j.ccm.2019.10.006.

[5] R.L. Siegel, K.D. Miller, H.E. Fuchs, A. Jemal, Cancer Statistics, 2021, CA: a cancer journal for clinicians 71(1) (2021) 7-33. https://doi.org/10.3322/caac.21654.

[6] G. Sartori, L. Belluomini, F. Lombardo, A. Avancini, I. Trestini, E. Vita, D. Tregnago, J. Menis, E. Bria, M. Milella, S. Pilotto, Efficacy and safety of afatinib for non-small-cell lung cancer: state-of-the-art and future perspectives, Expert Review of Anticancer Therapy 20(7) (2020) 531-542.

https://doi.org/10.1080/14737140.2020.1776119.

[7] T. Asao, F. Takahashi, K. Takahashi, Resistance to molecularly targeted therapy in non-small-cell lung cancer, Respiratory Investigation 57(1) (2019) 20-26. https://doi.org/10.1016/j.resinv.2018.09.001.

[8] P.O. Bolan, A. Zviran, L. Brenan, J.S. Schiffman, N. Dusaj, A. Goodale, F. Piccioni, C.M. Johannessen, D.A. Landau, Genotype-Fitness Maps of EGFR-Mutant Lung Adenocarcinoma Chart the Evolutionary Landscape of Resistance for Combination Therapy Optimization, Cell Systems 10(1) (2020) 52-65.e7. https://doi.org/10.1016/j.cels.2019.10.002.

[9] M. Xiang, H.G. Jiang, Y. Shu, Y.J. Chen, J. Jin, Y.M. Zhu, M.Y. Li, J.N. Wu, J. Li, Bisdemethoxycurcumin Enhances the Sensitivity of Non-small Cell Lung Cancer Cells to Icotinib via Dual Induction of Autophagy and Apoptosis, Int J Biol Sci 16(9) (2020) 1536-1550. https://doi.org/10.7150/ijbs.40042.

[10] J. Gao, H.R. Li, C. Jin, J.H. Jiang, J.Y. Ding, Strategies to overcome acquired resistance to EGFR TKI in the treatment of non-small cell lung cancer, Clinical and Translational Oncology 21(10) (2019) 12871301. https://doi.org/10.1007/s12094-019-02075-1. 
[11] D. Westover, J. Zugazagoitia, B.C. Cho, C.M. Lovly, L. Paz-Ares, Mechanisms of acquired resistance to first- and second-generation EGFR tyrosine kinase inhibitors, Ann Oncol 29(suppl_1) (2018) i10-i19. https://doi.org/10.1093/annonc/mdx703.

[12] S.P. Wheatley, D.C. Altieri, Survivin at a glance, Journal of cell science 132(7) (2019). https://doi.org/10.1242/jcs.223826.

[13] F. Shojaei, F. Yazdani-Nafchi, M. Banitalebi-Dehkordi, M. Chehelgerdi, M. Khorramian-Ghahfarokhi, Trace of survivin in cancer, European journal of cancer prevention : the official journal of the European Cancer Prevention Organisation (ECP) 28(4) (2019) 365-372.

https://doi.org/10.1097/CEJ.0000000000000453.

[14] S.S.J. W S Lin, J-T A Hsu,Shih Sheng Jiang ,Overcoming acquired resistance to BIBW2992 in HCC827, a non-small cell lung cancer cell line by dasatinib, arrayexpress-repository, V1, (2016).

[15] L. Girard, J. Rodriguezcanales, C. Behrens, D. Thompson, I. Botros, H. Tang, Y. Xie, N. Rekhtman, W.D. Travis, I.I. Wistuba, An expression signature as an aid to the histologic classification of non-small cell lung cancer, Clinical Cancer Research 22(19) (2016) 4880-4889. https://doi.org/10.1158/1078-0432.CCR15-2900.

[16] A. Sanchezpalencia, M. Gomezmorales, J.A. Gomezcapilla, V. Pedraza, L. Boyero, R. Rosell, M.E. Farezvidal, Gene expression profiling reveals novel biomarkers in nonsmall cell lung cancer, International Journal of Cancer 129(2) (2011) 355-364.

https://doi.org/ 10.1002/ijc.25704.

[17] T. Barrett, S.E. Wilhite, P. Ledoux, C. Evangelista, I.F. Kim, M. Tomashevsky, K.A. Marshall, K.H. Phillippy, P.M. Sherman, M. Holko, A. Yefanov, H. Lee, N. Zhang, C.L. Robertson, N. Serova, S. Davis, A. Soboleva, NCBI GEO: Archive for functional genomics data sets - Update, Nucleic Acids Research 41(D1) (2013) D991-D995. https://doi.org/10.1093/nar/gks1193.

[18] W. Huang da, B.T. Sherman, R.A. Lempicki, Systematic and integrative analysis of large gene lists using DAVID bioinformatics resources, Nature protocols 4(1) (2009) 44-57. https://doi.org/10.1038/nprot.2008.211.

[19] D.R. Rhodes, S. Kalyana-Sundaram, V. Mahavisno, R. Varambally, J. Yu, B.B. Briggs, T.R. Barrette, M.J. Anstet, C. Kincead-Beal, P. Kulkarni, S. Varambally, D. Ghosh, A.M. Chinnaiyan, Oncomine 3.0: Genes, Pathways, and Networks in a Collection of 18,000 Cancer Gene Expression Profiles, Neoplasia 9(2) (2007) 166-180. https://doi.org/ 10.1593/neo.07112.

[20] T. Li, J. Fan, B. Wang, N. Traugh, Q. Chen, J.S. Liu, B. Li, X.S. Liu, TIMER: A Web Server for Comprehensive Analysis of Tumor-Infiltrating Immune Cells, Cancer research 77(21) (2017) e108e110.https://doi.org/10.1158/0008-5472.CAN-17-0307. 
[21] Z. Tang, C. Li, B. Kang, G. Gao, C. Li, Z. Zhang, GEPIA: a web server for cancer and normal gene expression profiling and interactive analyses, Nucleic Acids Res 45(W1) (2017) W98-w102. https://doi.org/10.1093/nar/gkx247.

[22] A. Lánczky, Á. Nagy, G. Bottai, G. Munkácsy, A. Szabó, L. Santarpia, B. Győrffy, miRpower: a web-tool to validate survival-associated miRNAs utilizing expression data from 2178 breast cancer patients, Breast cancer research and treatment 160(3) (2016) 439-446. https:// 10.1007/s10549-016-4013-7.

[23] D. Warde-Farley, S.L. Donaldson, O. Comes, K. Zuberi, R. Badrawi, P. Chao, M. Franz, C. Grouios, F. Kazi, C.T. Lopes, A. Maitland, S. Mostafavi, J. Montojo, Q. Shao, G. Wright, G.D. Bader, Q. Morris, The GeneMANIA prediction server: biological network integration for gene prioritization and predicting gene function, Nucleic Acids Research 38(suppl_2) (2010) W214-W220. https://doi.org/10.1093/nar/gkq537.

[24] A. Benito-Martin, H. Peinado, FunRich proteomics software analysis, let the fun begin!, PROTEOMICS 15(15) (2015) 2555-2556. https:// 10.1002/pmic.201500260.

[25] N.K. Lytle, A.G. Barber, T.J.N.R.C. Reya, Stem cell fate in cancer growth, progression and therapy resistance, 18(11) (2018) 669-680. https://doi.org/10.1038/s41568-018-0056-x.

[26] Z.F. Lim, P.C. Ma, Emerging insights of tumor heterogeneity and drug resistance mechanisms in lung cancer targeted therapy, Journal of hematology \& oncology 12(1) (2019) 134. https:// doi.org/ 10.1186/s13045-019-0818-2.

[27] M. Haschka, G. Karbon, L.L. Fava, A. Villunger, Perturbing mitosis for anti-cancer therapy: is cell death the only answer?, EMBO reports 19(3) (2018). https:// doi.org/10.15252/embr.201745440.

[28] C. Buccitelli, M. Selbach, mRNAs, proteins and the emerging principles of gene expression control, Nature Reviews Genetics 21(10) (2020) 630-644. https:// doi.org/10.1038/s41576-020-0258-4.

[29] J. Han, L.A. Goldstein, W. Hou, S. Chatterjee, T.F. Burns, H. Rabinowich, HSP90 inhibition targets autophagy and induces a CASP9-dependent resistance mechanism in NSCLC, Autophagy 14(6) (2018) 958-971. https:// doi.org/10.1080/15548627.2018.1434471.

[30] J. Bertranalamillo, V. Cattan, M. Schoumacher, J. Codonyservat, A. Gimenezcapitan, F. Cantero, M.F. Burbridge, S. Rodriguez, C. Teixido, R.J.N.C. Roman, AURKB as a target in non-small cell lung cancer with acquired resistance to anti-EGFR therapy, 10(1) (2019) 1812. https://doi.org/10.1038/s41467-019-097345 .

[31] K.N. Shah, R. Bhatt, J. Rotow, J. Rohrberg, V. Olivas, V. Wang, G. Hemmati, M.M. Martins, A. Maynard, J.J.N.M. Kuhn, Aurora kinase A drives the evolution of resistance to third-generation EGFR inhibitors in lung cancer, 25(1) (2019) 111-118. https://doi.org/10.1038/s41591-018-0264-7.

[32] P. Khongkow, A.R. Gomes, C. Gong, E.P.S. Man, J.W.H. Tsang, F. Zhao, L.J. Monteiro, R.C. Coombes, R.H. Medema, U.S.J.O. Khoo, Paclitaxel targets FOXM1 to regulate KIF20A in mitotic catastrophe and 
breast cancer paclitaxel resistance, 35(8) (2016) 990-1002. https://doi.org/ 10.1038/onc.2015.152.

[33] B. Zhao, J. Zhang, X. Chen, H. Xu, B.J.A.o.M.S. Huang, Mir-26b inhibits growth and resistance to paclitaxel chemotherapy by silencing the CDC6 gene in gastric cancer, 15(2) (2019) 498-503. https://doi.org/ 10.5114/aoms.2018.73315.

[34] J.A. Hellyer, H. Stehr, M. Das, S.K. Padda, K. Ramchandran, J.W. Neal, M. Diehn, H.A.J.L.C. Wakelee, Impact of KEAP1/NFE2L2/CUL3 mutations on duration of response to EGFR tyrosine kinase inhibitors in EGFR mutated non-small cell lung cancer, 134 (2019) 42-45. https://doi.org/ 10.1016/j.lungcan.2019.05.002.

[35] R. Niu, Y. Tang, Y. Xi, D.J.J.o.S.R. Jiang, High Expression of Krüppel-like Factor 7 Indicates Unfavorable Clinical Outcomes in Patients with Lung Adenocarcinoma, 250 (2020) 216-223. https://doi.org/10.1016/j.jss.2019.12.053.

[36] Y. An, Y. Shang, Z. Xu, Q. Zhang, Z. Wang, W. Xuan, X.J.B. Zhang, Pharmacotherapy, STAT3-induced long noncoding RNA LINC00668 promotes migration and invasion of non-small cell lung cancer via the miR-193a/KLF7 axis, 116 (2019) 109023. https://doi.org/10.1016/j.biopha.2019.109023.

[37] W.-j. Liu, Y. Du, R. Wen, M. Yang, J. Xu, Drug resistance to targeted therapeutic strategies in non-small cell lung cancer, Pharmacology \& Therapeutics 206 (2020) 107438. https://doi.org/10.1016/j.pharmthera.2019.107438.

[38] S. Kohsaka, M. Petronczki, F. Solca, M.J.F.O. Maemondo, Tumor clonality and resistance mechanisms in EGFR mutation-positive non-small-cell lung cancer: implications for therapeutic sequencing, 15(6) (2019) 637-652. https://doi.org/10.2217/fon-2018-0736.

[39] T. Ito, Y. Kumagai, K. Itano, T. Maruyama, K. Tamura, S. Kawasaki, T. Suzuki, Y. Murakami, Mathematical analysis of gefitinib resistance of lung adenocarcinoma caused by MET amplification, Biochemical and Biophysical Research Communications 511 (3) (2019) 544-550. https://doi.org/10.1016/j.bbrc.2019.02.086.

[40] D. Westover, J. Zugazagoitia, B.C. Cho, C.M. Lovly, L. Paz-Ares, Mechanisms of acquired resistance to first- and second-generation EGFR tyrosine kinase inhibitors, Ann Oncol 29(suppl_1) (2018) i10-i19. https://doi.org/10.1093/annonc/mdx703.

[41] M. Metzenmacher, D.C. Christoph, Efficacy and safety of nivolumab combined with standard therapies for first-line therapy of advanced non-small cell lung cancer, Journal of Thoracic Disease; Vol 8 , No 10 (October 2016): Journal of Thoracic Disease (2016). https://doi.org/10.21037/jtd.2016.10.26.

[42] B. Ricciuti, S. Baglivo, A. De Giglio, R.J.T.A.i.R.D. Chiari, Afatinib in the first-line treatment of patients with non-small cell lung cancer: clinical evidence and experience, 12 (2018) 1753466618808659. https://doi.org/ 10.1177/1753466618808659. 
[43] D. Westover, J. Zugazagoitia, B.C. Cho, C.M. Lovly, L.J.A.o.O. Pazares, Mechanisms of acquired resistance to first- and second-generation EGFR tyrosine kinase inhibitors, 29 (2018). https://doi.org/ 10.1093/annonc/mdx703.

[44] F. Nasim, B.F. Sabath, G.A. Eapen, Lung Cancer, The Medical clinics of North America 103(3) (2019) 463-473. https://doi.org/ 10.1016/j.mcna.2018.12.006.

[45] R. Arafeh, Y. Samuels, PIK3CA in cancer: The past 30 years, Seminars in Cancer Biology 59 (2019) 3649. https://doi.org/10.1016/j.semcancer.2019.02.002.

[46] S.P. Wheatley, D.C. Altieri, Survivin at a glance, Journal of cell science 132(7) (2019) jcs223826. https://doi.org/10.1242/jcs.223826.

[47] G. Zhao, Q. Wang, Z. Wu, X. Tian, H. Yan, B. Wang, P. Dong, H. Watari, L.M. Pfeffer, Y. Guo, W. Li, J. Yue, Ovarian primary and metastatic tumors suppressed by survivin knockout or a novel survivin inhibitor, Molecular Cancer Therapeutics 18(12) (2019) 2233-2245. https://doi.org/10.1158/1535-7163.MCT-190118.

[48] S.P. Wheatley, D.C.J.J.o.C.S. Altieri, Survivin at a glance, 132(7) (2019). https://doi.org/10.1242/jcs.223826.

[49] T. Shimizu, K. Nishio, K. Sakai, I. Okamoto, K. Okamoto, M. Takeda, M. Morishita, K. Nakagawa, Phase I safety and pharmacokinetic study of YM155, a potent selective survivin inhibitor, in combination with erlotinib in patients with EGFR TKI refractory advanced non-small cell lung cancer, Cancer Chemother Pharmacol 86(2) (2020) 211-219. https://doi.org/10.1007/s00280-020-04112-1.

[50] R.C. Peery, J.-Y. Liu, J.-T. Zhang, Targeting survivin for therapeutic discovery: past, present, and future promises, Drug Discovery Today 22(10) (2017) 1466-1477. https://doi.org/10.1016/j.drudis.2017.05.009.

[51] F. Li, I. Aljahdali, X. Ling, Cancer therapeutics using survivin BIRC5 as a target: what can we do after over two decades of study?, Journal of Experimental \& Clinical Cancer Research 38(1) (2019) 368. https://doi.org/10.1186/s13046-019-1362-1.

[52] M. De Donato, G. Babini, S. Mozzetti, M. Buttarelli, A. Ciucci, G. Arduini, M.C. De Rosa, G. Scambia, D. Gallo, KLF7: a new candidate biomarker and therapeutic target for high-grade serous ovarian cancer, Journal of Experimental \& Clinical Cancer Research 39(1) (2020) 265. ical Cancer Research (2020) 39:265. https://doi.org/10.1186/s13046-020-01775-9.

[53] R. Niu, Y. Tang, Y. Xi, D. Jiang, High Expression of Krüppel-like Factor 7 Indicates Unfavorable Clinical Outcomes in Patients with Lung Adenocarcinoma, The Journal of surgical research 250 (2020) 216-223. https://doi.org/ 10.1016/j.jss.2019.12.053.

\section{Figures}



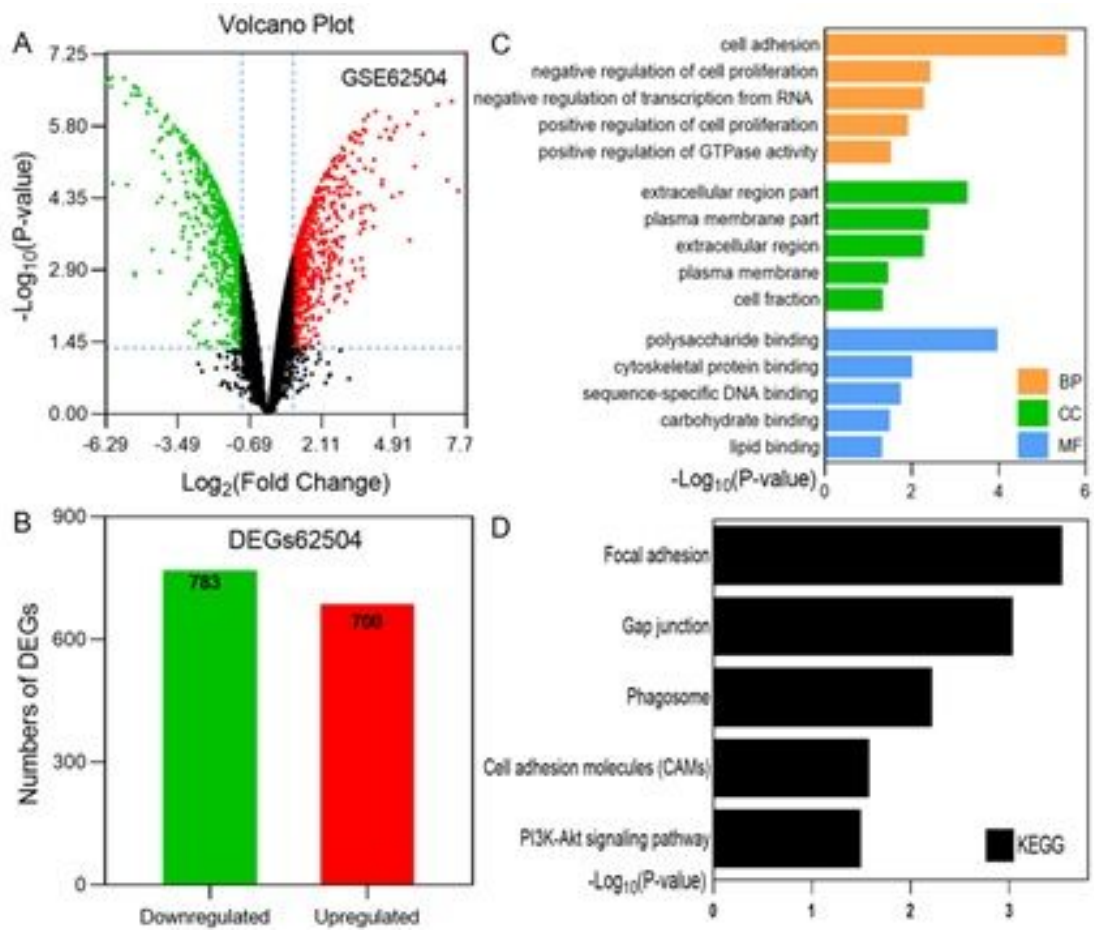

Figure 1

Identification and characterization of DEGs from the GSE62504 dataset. (A) Volcano plot of DEGs between afatinib-resistant cells and parental cells. Red dots indicate significantly upregulated DEGs in afatinib-resistant cells, green dots, afatinib-resistant DEGs downregulated; black dots, no significant difference ( $P<0.05$ and $|\log 2 F C|>2$ as the threshold). (B) DEG distribution of significance in afatinibresistant cells. The top $5 \mathrm{GO}$ terms (C) and KEGG enriched pathways (D) of significantly upregulated DEGs in NSCLC tissues are indicated. BP, biological process; CC, cell component; MF, molecular function. 



Figure 2

Identification and characterization of DEGs from the GSE75037 dataset. (A) Volcano plot of DEGs between NSCLC tissues and adjacent normal tissues. Red dots are significantly upregulated DEGs in NSCLC tissues; green dots, DEGs downregulated in NSCLC tissues; black dots, no significant difference $(\mathrm{P}<0.05$ and $|\log 2 \mathrm{FC}|>2$ as the threshold). (B) DEGs distribution of significant in NSCLC tissues. The top 5 GO terms (C) and KEGG enriched pathways (D) of significantly upregulated DEGs in NSCLC tissues are indicated. BP, biological process; CC, cell component; MF, molecular function. 

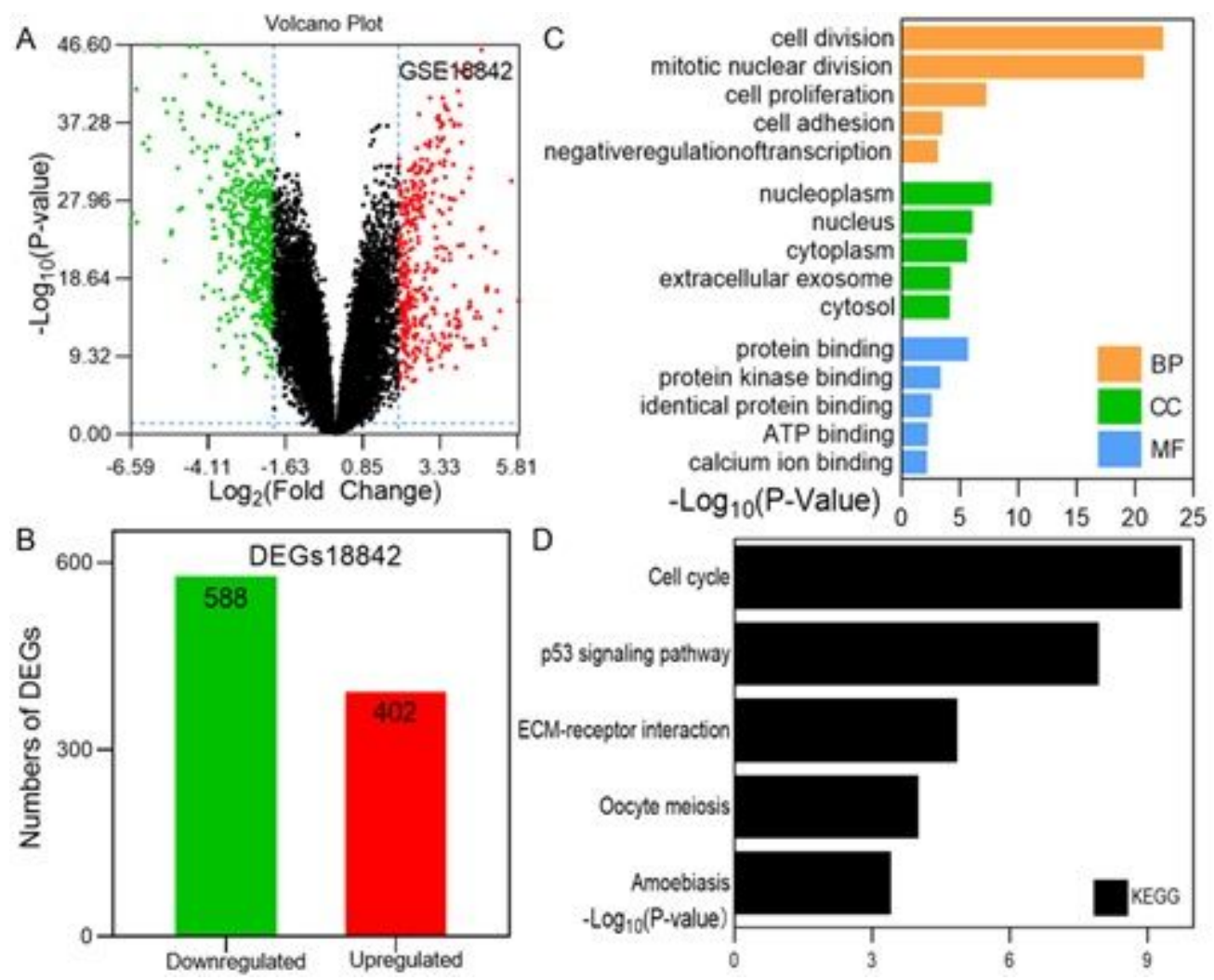

\section{Figure 3}

Identification and characterization of DEGs from the GSE18842 dataset. (A) Volcano plot of DEGs between NSCLC tissues and adjacent normal tissues. Red dots are significantly upregulated DEGs in NSCLC tissues; green dots, DEGs downregulated in NSCLC tissues; black dots, no significant difference $(\mathrm{P}<0.05$ and $|\log 2 \mathrm{FC}|>2$ as the threshold). (B) DEG distribution of significance in NSCLC tissues. The top 5 GO terms (C) and KEGG enriched pathways (D) of significantly upregulated DEGs in NSCLC tissues are indicated. BP, biological process; CC, cell component; MF, molecular function. 

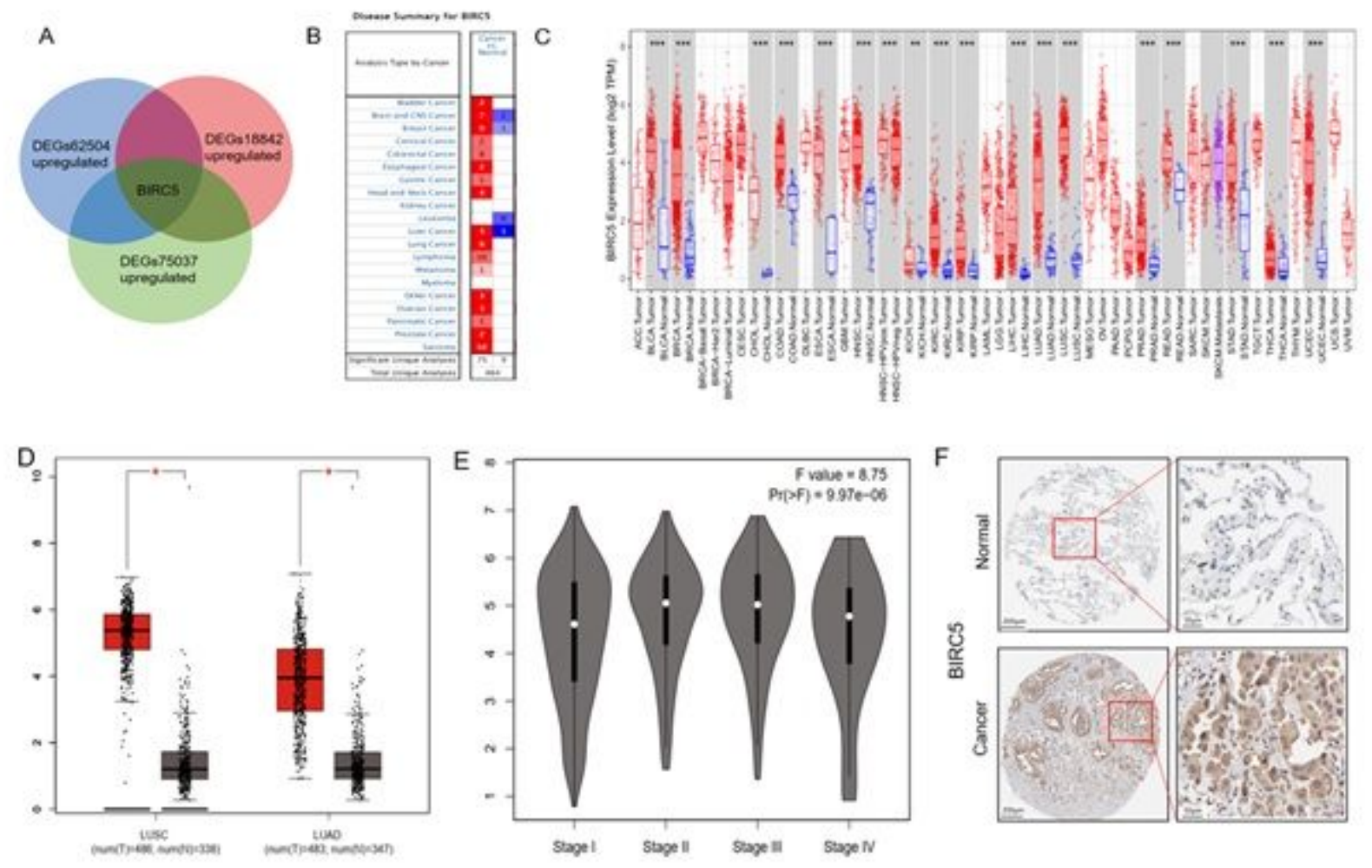

Figure 4

Verification of BIRC5's afatinib expression function. (A) Venn diagram of significantly upregulated DEGs from the GSE62504, GSE75037 and GSE18842 datasets. (B) The expression level of BIRC5 in different cancers compared with normal tissues in the Oncomine database (P区.0001, $|\mathrm{FC}|>2$, and gene ranking of all.). (C) Human BIRC5 expression levels in different tumor types from the TCGA database were determined by TIMER (*P囚.05, ${ }^{*} \mathrm{P} \bigotimes .01,{ }^{* * * P \bigotimes .001)}$. (D) The expression of BIRC5 in NSCLC patients (GEPIA). Box plots derived from gene expression data for GEPIA comparing the expression of BIRC5 in NSCLC tissue and normal tissues; the p-value was set at 0.05. (E) Correlation between BIRC5 mRNA expression and tumor stage in lung cancer patients by GEPIA. Violin plot derived from the correlation between the expression of BIRC5 and tumor stage in patients with lung cancer; the p-value was set at 0.05. (F) Immunohistochemical staining of BIRC5 protein expression in normal lung tissue and NSCLC tissue was obtained from the Human Protein Atlas online database (magnification, $\times 40$ ). 

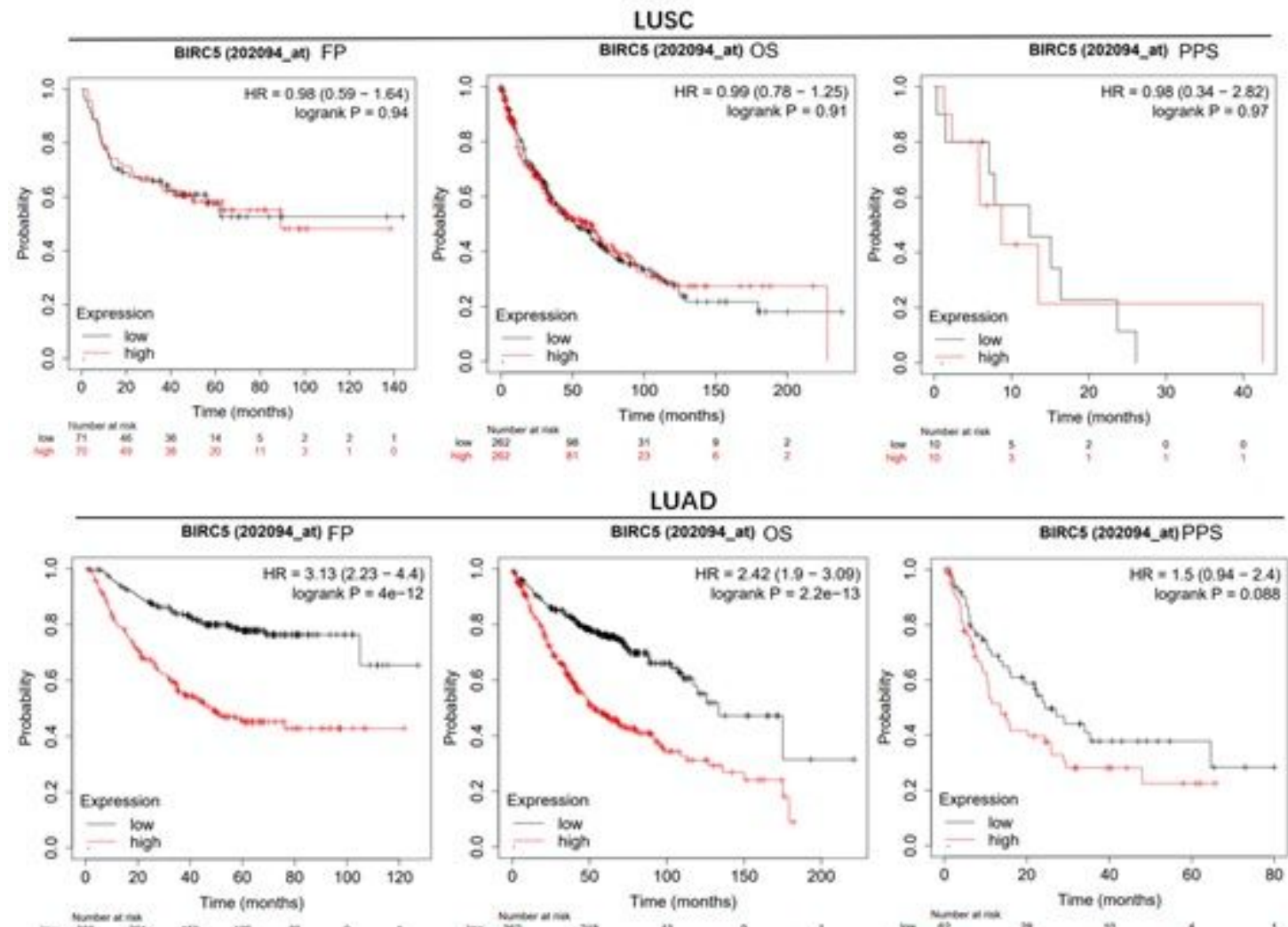

$\Rightarrow \operatorname{lom}_{\infty}^{\infty}$

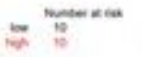

LUAD
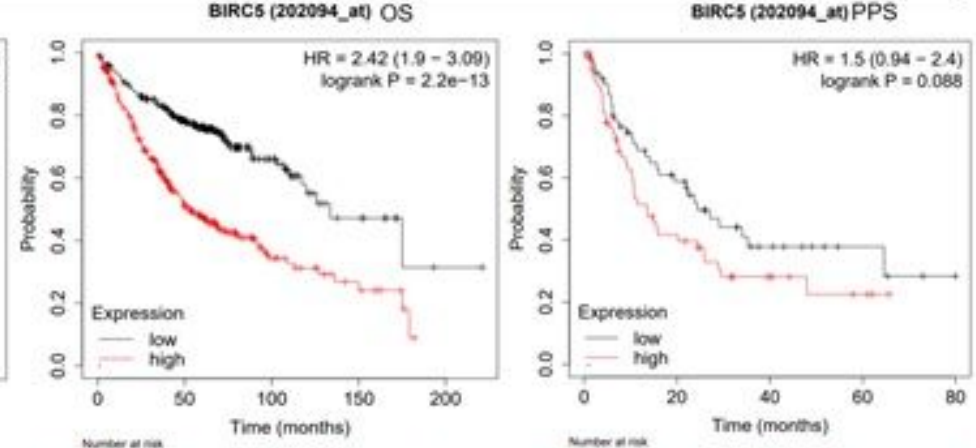

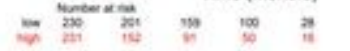

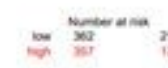

\section{Figure 5}

Survival analysis FP, OS and PPS of BIRC5 in LUSC and LUAD patients obtained from KM plotter.

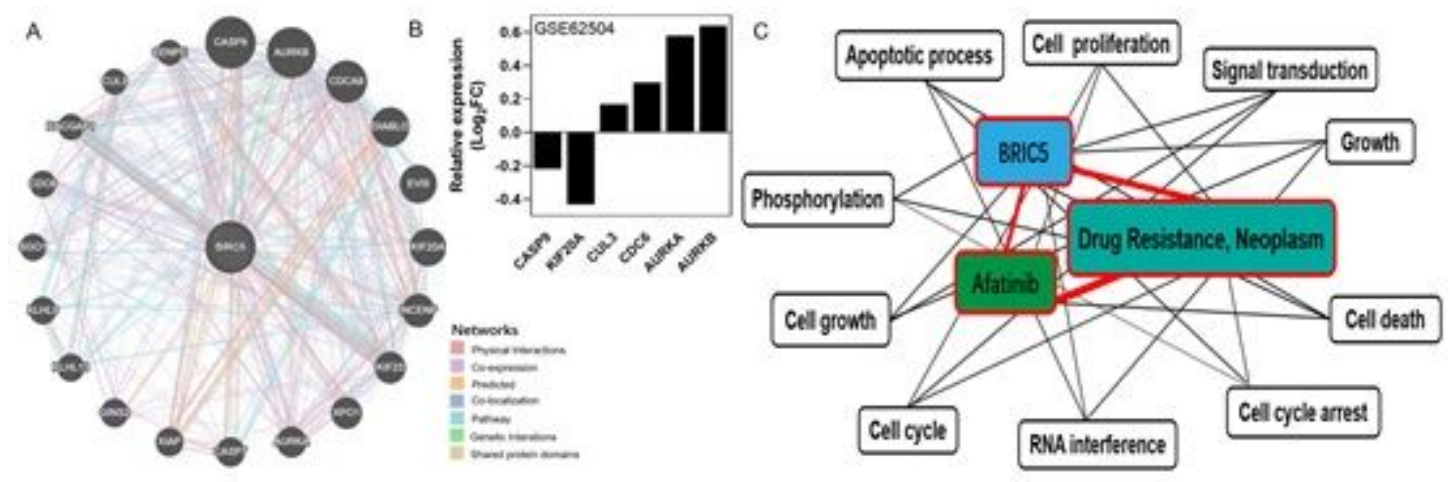

Figure 6

Validation of the afatinib resistance function of BIRC5. (A) Protein/gene-protein/gene interaction network of BIRC5 produced by GeneMANIA. (B) Relative mRNA levels of 5 genes interacting with BIRC 5 based on the microarray data of GSE62504. (C) Annotation of biological processes of BIRC5 in NSCLC and drug resistance using Coremine Medical software. 